Increased weight and, more recently, body mass index (BMI), have been suggested as risk factors for carpal tunnel syndrome (CTS). In an effort to determine the relative risk (RR) of obesity in the development of CTS, 949 patients who had an evaluation of the right upper extremity that included motor and sensory conduction studies of the median and ulnar nerves were reviewed. Of these patients, 261 were diagnosed with a median mononeuropathy at the wrist. Those individuals who were classified as obese $(B M I>29)$ were 2.5 times more likely than slender individuals $(\mathrm{BMI}<20)$ to be diagnosed with CTS. Forty-three percent of obese women and $32 \%$ of obese men had the diagnosis of CTS compared to $21 \%$ of slender women and $0 \%$ of slender men. (c) 1994 John Wiley \& Sons, inc.

Key words: body mass index - carpal tunnel syndrome - median nerve injury • electromyography $\cdot$ relative risk

MUSCLE \& NERVE 17:632-636 1994

\title{
THE RELATIONSHIP BETWEEN BODY MASS INDEX AND THE DIAGNOSIS OF CARPAL TUNNEL SYNDROME
}

\author{
ROBERT A. WERNER, MD, JAMES W. ALBERS, MD, PhD, \\ ALFRED FRANZBLAU, MD, and THOMAS J. ARMSTRONG, PhD
}

There are many factors thought to place an individual at risk for the development of carpal tunnel syndrome (CTS). Numerous personal cofactors (see Table 1) have been reported but very few have been quantified as to the strength of the association. $^{2,4,5}$ Several investigators have reported that individuals with CTS were heavier and shorter than the general population. ${ }^{3,5,6,11,16,17}$ Cannon $^{3}$ noted that $27 \%$ of individuals ( 8 of 30 ) with CTS were obese compared to $12 \%$ (11 of 90 ) in a control population; this difference did not reach statistical significance. Falck and Aarnio ${ }^{6}$ described 17 butchers, most of whom were obese, and found that $53 \%$ had CTS. There was no difference in the body mass index (BMI) between butchers with or without CTS. Dieck and Kelsey ${ }^{5}$ found an in-

From the Department of Physical Medicine and Rehabilitation, University of Michigan Medical Center. Ann Arbor, Michigan (Drs. Werner and Albers); Physical Medicine and Rehabilitation Service, Veterans Affairs Medical Center, Ann Arbor, Michigan (Drs. Werner and Albers): Department of Neurology, University of Michigan Medical Center, Ann Arbor, Michigan (Dr. Albers); and Department of Environmental and Industrial Health, School of Public Health, University of Michigan, Ann Arbor, Michigan (Drs. Franzblau and Armstrong).

Address reprint requests to Robert A. Werner, MD, $1500 \mathrm{E}$. Medical Center Drive, Box 0042, University Hospital, Ann Arbor, MI 48109-0042

Acknowledgment: We thank Lincoln Jaros for his help in establishing this data set and the development of the EMGpro software.

Accepted for publication December 17, 1993

CCC 0148-639//94/060632-05

(C) 1994 John Wiley \& Sons, Inc creased prevalence of CTS, within an adult female population, among individuals with short stature, greater weight and recent weight gain. The BMI was significantly higher in the CTS group $(27 \mathrm{~kg} /$ $\mathrm{m}^{2}$ vs. $25 \mathrm{~kg} / \mathrm{m}^{2}, P=0.01$ ). Vessey et al. ${ }^{15}$ found that the risk for CTS among obese women was double that of slender women.

Within an industrial population, Nathan et al. ${ }^{11}$ demonstrated that a higher BMI was associated with a higher prevalence of median mononeuropathy. They found a relative risk (RR) of 4.1 for obese individuals compared to slender individuals. This relationship was more pronounced in men $(R R=5.1)$ than in women $(R R=2.7)$. This study did have a number of methodological flaws of which the most prominent was an analysis by hand instead of by person.

Most prior studies were relatively small and primarily addressed the relationship between CTS and obesity among women. Nathan's study was larger and included both genders but surveyed an industrial-based population. The purpose of this study was to determine the risk of obese individuals for the development of CTS in a large nonindustrial population.

\section{METHODS}

A cross-sectional study design was employed to study the relative risk for CTS related to body size. Of approximately 6000 patients seen in the elec- 
Table 1. Personal cofactors related to the occurrence of carpal tunnel syndrome.

Medical conditions
Diabetes
Thyroid disease
Connective tissue disorders
Amyloidosis
Acromegalia
Vitamin $\mathrm{B}_{6}$ deficiency (?)
Age
Gender
Oral contraceptive use
Oophorectomy
Wrist dimension
Weight
Stature
Body mass index (BMI)

trodiagnostic laboratory during 1991 and 1992, 949 patients were identified who were: (1) referred for evaluation of the right upper extremity; (2) completed an evaluation of the median and ulnar, motor and sensory, nerves; and (3) reported height, weight, and age. The diagnosis of a median mononeuropathy was based on the relative difference in peak sensory latency between the median and ulnar responses $(14 \mathrm{~cm}$, antidromic stimulation) using a threshold of abnormality of $0.5 \mathrm{~ms}$ or longer. ${ }^{9}$ The standard within our laboratory dictated that if there was a clinical suspicion and the standard sensory evoked responses were borderline, median and ulnar midpalmar responses were obtained; a difference of $0.3 \mathrm{~ms}$ or greater was considered diagnostic of a median mononeuropathy. If the median sensory responses were absent, median motor latency was compared to ulnar motor latency with a 1.7-ms difference being defined as abnormal. All subjects had midpalm temperatures recorded and were warmed if the temperature was below $32.0^{\circ} \mathrm{C}$. The combination of clinical symptoms and a median mononeuropathy was defined as CTS. All further reference to CTS among subjects in this study will use this case definition.

The BMI $\left(\mathrm{kg} / \mathrm{m}^{2}\right)$ was calculated for each subject and subgroups were created based upon the BMI distribution reported for the state of Michigan: slender, BMI < 20; normal, BMI 20-25; heavy, BMI 25-29; and obese, BMI > 29. ${ }^{1}$ Age was also divided into three age groups: (1) less than 45 ; (2) 45-64; and (3) 65 or older.

\section{STATISTIGS}

Statistical analysis was done using STATA. ${ }^{13}$ Preliminary analysis consisted of $2 \times K$ tables of diag- nosis (CTS and "other") by body size (slender, normal, heavy, and obese), age group, and gender. Pearson chi-squared statistics were used to determine the variation from expected values. Risk ratios were determined for body size, age group, and gender. Mantel-Haenszel statistics were used to evaluate for interactions between the independent variables. Logistic regressions were performed using the diagnosis of CTS versus "other" as the dependent variable and age, gender, and BMI as the independent variables. Age was analyzed as a continuous as well as an ordinal variable as was BMI. Interactions as well as second and third order variables were tested in the model. Finally, multiple linear regressions were developed with the difference between the median and ulnar sensory latencies as the dependent variable and age, gender, and BMI as the independent variables. The same statistical analyses were performed using a subset of the population; individuals with CTS (but no other codiagnosis) were compared to individuals with a normal study. All results are reported as a mean and 1 standard deviation [mean (l SD)]. All risk ratios or odds ratios are presented with the 95\% confidence interval following in parentheses. Any interval that includes the number 1.0 is not significant at the $P=0.05$ level.

\section{RESULTS}

Of the 949 subjects, the average age was 46.1 (14.7) with $56 \%$ being female. The mean BMI was 26.8 (6.4) with $28.6 \%$ being classified as obese, $27.8 \%$ as heavy, $35.6 \%$ as normal, and $8 \%$ as slender. Two hundred sixty-one subjects $(27.5 \%)$ were diagnosed with CTS, while $342(36 \%)$ were characterized as normal, with the remaining $36.5 \%$ of subjects having some other electrodiagnostic abnormality (i.e., cervical radiculopathy, brachial plexopathy, ulnar neuropathy, peripheral polyneuropathy, etc.).

Figure 1 demonstrates the relationship between body size and the percent of patients diagnosed with CTS. Only $16 \%$ of slender individuals were diagnosed with CTS compared to $39 \%$ of obese subjects. The relative risk for obese individuals compared to slender individuals, without controlling for other variables, is $2.5(95 \% \mathrm{CI}=1.4,4.2)$. This means obese individuals are 2.5 times as likely to have the diagnosis of CTS compared to a group of slender individuals. The mean BMI for those individuals with CTS was $28.9(6.8)$ and $26.2(6.0)$ in the remainder of the population. The distribution of BMI for each diagnostic group is presented 


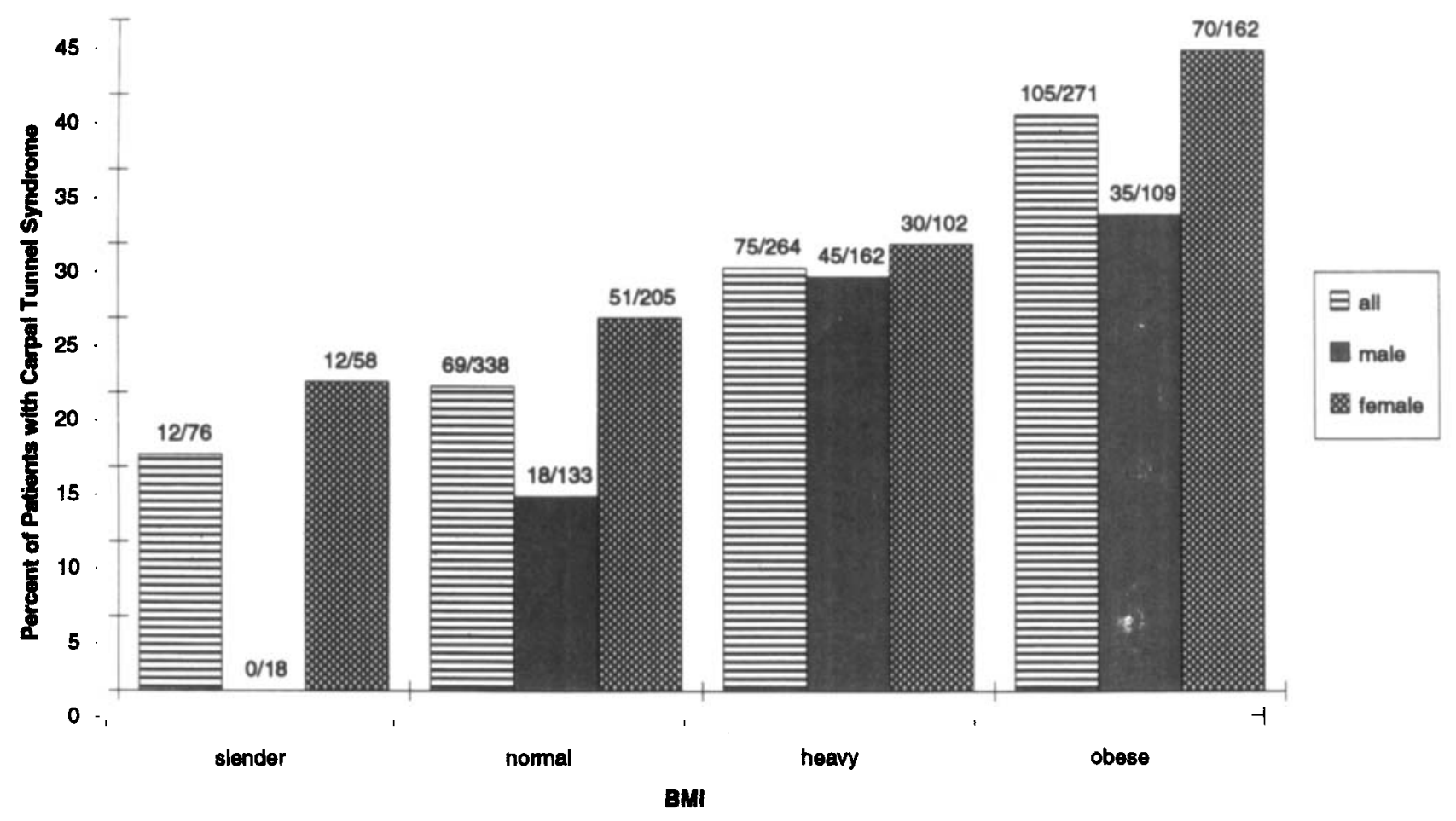

FIGURE 1. The percent of patients with carpal tunnel syndrome by body type: slender (BMI <20), normal (BMI 20-25), heavy (BMI 25-29), obese $(\mathrm{BMI}>29)$.

graphically in Figure 2. These populations are statistically different at $P<0.001$.

Gender had an influence on the diagnosis of CTS. Thirty-one percent of females were diagnosed with CTS compared to $23 \%$ of males. There was an apparent interaction between body size and gender with respect to the diagnosis of CTS. Obese men had a RR of $11.7(95 \% \mathrm{CI}=2.5,55.6)$ compared to slender men. This was in contrast to a RR of $2.1(95 \% \mathrm{CI}=1.2,3.6)$ for obese women. This relationship is presented in Figure 1. The prevalence of CTS was highest in obese women and lowest in thin and normal-sized men.

The mean age of the CTS group was 48.1 (14.1) years compared to 44.7 (15.7) years in the rest of the population ( $t$-test, $P=0.001$ ). There was a peak prevalence in the age group 45-65 (41\%) followed by the subjects over $65(26 \%)$ and the lowest prevalence in the portion of the population under 45 (21\%).

Correlation coefficients were determined between BMI, age, median sensory latency, and ulnar sensory latency. There was a significant correlation between BMI and the median sensory latency $(r=0.12, P<0.001)$ but it was not significant between BMI and ulnar nerve responses $(r=$ $0.05, P=0.11$ ). The difference between the median and ulnar sensory latencies demonstrated a stronger correlation $(r=0.22, P<0.001)$. Age was correlated with both median and ulnar sensory latencies $(r=0.31$ and 0.24 , respectively; $P<$ $0.001)$. Age and BMI were not significantly correlated $(r=0.03, P=0.33)$.

In order to explore the contribution of each of the independent variables while controlling for the effect of the other variables, as well as looking for second order effects or interactions, a logistic regression analysis was performed. This analysis gives an odds ratio which is an estimate of the relative risk. The dependent variable was the diagnosis of CTS versus "other." All three independent variables, body size, age, and gender, were significant when controlling for the others but no second order variable or interaction term was significant. The odds ratio of obese compared to slender individuals was $8.2(95 \% \mathrm{CI}=1.1,63.0)$ but when overweight and obese individuals were compared to slender and normal individuals the odds ratio was $2.9(95 \% \mathrm{CI}=1.6,5.0)$. Individuals aged $45-$ 65 were $2.0(95 \% \mathrm{CI}=1.4,2.7)$ times as likely to have been diagnosed with CTS compared to individuals under 45 years old. Those individuals over 65 years were $1.4(95 \% \mathrm{CI}=0.9,2.2)$ times as likely to have CTS compared to the under- 45 group, but this is not considered significant at the $P=0.05$ level. Women were at $1.5(95 \% \mathrm{CI}=1.1$, 

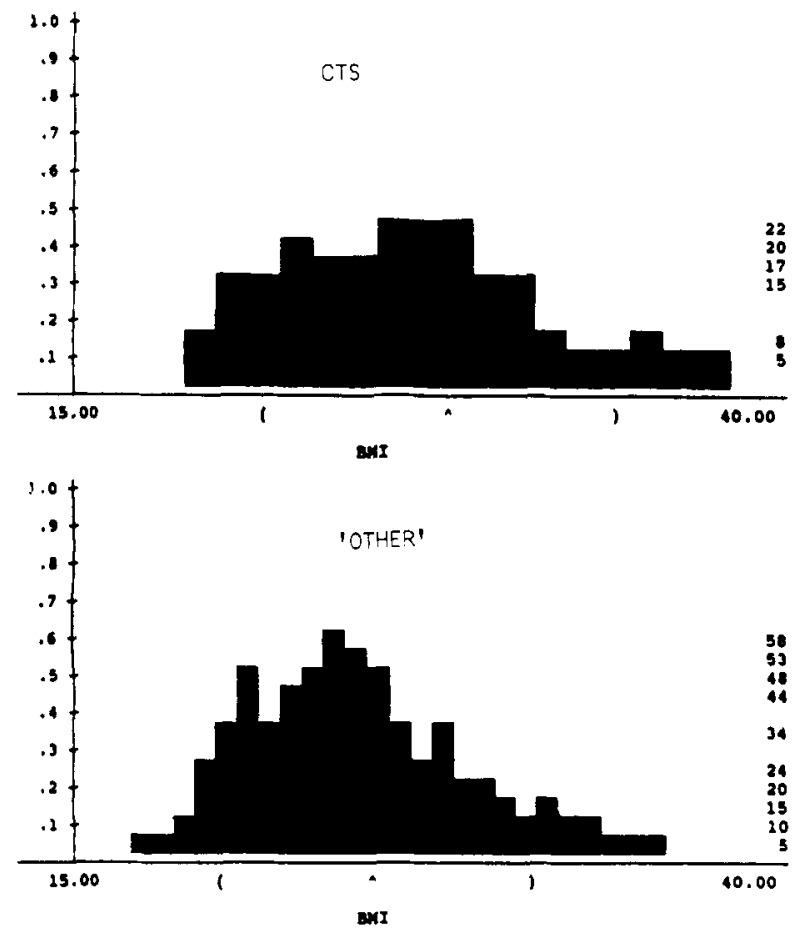

FIGURE 2. The distribution of body mass index (BMI) among patients with a diagnosis of carpal tunnel syndrome versus patients with arm/hand complaints and no evidence of CTS on nerve conduction studies ("other"). ( ${ }^{\wedge}$ ) represents the mean and 1 standard deviation. The scale on the right border represents the number of patients represented in the histogram.

2.1) times the risk for getting CTS compared to men. The apparent relationship between gender and slender body size was not significant. This most likely reflects the small number of the slender men in the population $(n=18)$. There was a trend suggesting that slender men were protected against CTS, but the sample size of this subgroup was small and the finding was not statistically significant.

The multiple linear regression model with the difference between median and ulnar sensory latencies as the dependent variable demonstrated that BMI, age, and gender were significant but only accounted for $8 \%$ of the total variance. Within this model, BMI was the most influential variable accounting for $5 \%$ of the variance; age and gender accounted for the other $2.6 \%$ explained in the model. Second order variables and interactions were not significant.

A secondary analysis was done on a subset of the population that had a diagnosis of CTS but no other electrodiagnostic abnormality and compared them to individuals classified as normal. The findings were very similar to the population as a whole with the exception that gender was not a significant factor in the analysis.

\section{DIscussion}

The findings of this study support the hypothesis that individuals with a higher BMI are at increased risk for CTS. The pathophysiology that would explain this relationship is not well understood. These observations support the findings of Nathan et al., ${ }^{11}$ as well as Vessey et al., ${ }^{15}$ but still explain only a small portion of the variance related to the diagnosis of CTS or electrodiagnostic abnormalities involving the median nerve. This study also reaffirms that the highest prevalence of CTS is in the age group 45-65 years and that women are at a higher risk than men for CTS. ${ }^{14}$

If a causal relationship between obesity and a slowing of median conduction across the wrist exists, it may relate to increased fatty tissue within the carpal canal or to increased hydrostatic pressure throughout the carpal canal in obese individuals compared to normal or slender individuals. Magnetic resonance imaging has demonstrated increased fatty tissue in the carpal canal being associated with CTS in 1 patient, ${ }^{10}$ but there has not been a systematic study of the intracarpal canal anatomy in obese compared to slender individuals. Likewise, intracarpal canal pressures have been demonstrated to be higher in individuals with CTS, $, 8,12,16$ but the relationship between body size and intracarpal canal pressure has not been explored.

The finding that BMI is correlated with the median but not ulnar sensory distal latencies suggest that the condition of obesity affects the nerves differently. The additional finding that the difference in the latencies is more strongly correlated with BMI than the median latency alone further supports this contention. The median nerve at the wrist is more compartmentalized than the ulnar nerve and may be subjected to compression due to fatty build up within the carpal canal among obese individuals. Conversely, heavier individuals may simply place more mechanical stress on their hands and wrists and thus place the median nerve at higher risk as opposed to some intrinsic change within the carpal canal.

The finding that women were more likely to have a higher prevalence of CTS than men supports other population based studies ${ }^{14}$ but differs from the worker compensation-based data on CTS reported by Franklin. ${ }^{7}$ In the workplace, the risk for women is only $10-20 \%$ higher than men as 
opposed to $300 \%$ reported in population-based studies. ${ }^{7,14}$

The population reported here, from a hospitalbased EMG laboratory, does not represent the general population and is substantially different from the industrial-based survey studied by Nathan. ${ }^{11}$ The fact that BMI was a significant risk factor in both studies strengthens its importance as a risk factor for CTS. Also this is not an association that is unique to women. Despite the prevalence of CTS being higher in women, obesity seems to be a more significant risk factor for men in our study as well as Nathan's. Conversely, being slender may be a protective factor with this being most striking in men. This was a retrospective study and was not able to factor in the role of exposure to cumulative trauma or to define the influence of the concurrent medical diagnoses on the prevalence of CTS. The possible association between obesity and the development of type II diabetes may be a confounder that we are unable to assess in this study.

Additionally, caution must be used in applying this information in the clinical setting. As demonstrated by Figure 1, not all obese individuals develop CTS. Sixty-five percent of the obese individuals in this study did not have CTS. Conversely, $20 \%$ of thin and normal individuals did develop CTS. The mechanism underlying this relationship is not understood and causation cannot be determined.

\section{REFERENCES}

1. Anderson JV, Rafferty AP: Overweight, in Health Risk Behaviors-1991. Lansing, MI, Michigan Department of Public Health, 1992.

2. Armstrong TJ, Chaffin DB: Carpal tunnel syndrome and selected personal attributes. J Occup Med 1979;21:481-486.

3. Cannon LJ, Bernacki EJ, Walter DS: Personal and occupational factors associated with carpal tunnel syndrome. $J O c^{-}$ cup Med 1982;23:255-258.

4. de Krom MC, Kester AD, Knipschild PG, Spanns F: Risk factors for carpal tunnel syndrome. Am J Epidemiol 1990; 132:1102-1110.

5. Dieck GS, Kelsey JL: An epidemiologic study of the carpal tunnel syndrome in an adult female population. Prev Med $1985 ; 14: 63-69$

6. Falck B, Aarnio P: Left-sided carpal tunnel syndrome in butchers. Scand J World Environ Health 1983;9:291-297.

7. Franklin GM, Haug J, Heyer N, Checkoway H, Peck N: Occupational carpal tunnel syndrome in Washington State, 1984-1988. Am J Public Health 1991;82:741-746.

8. Gelberman RC, Hergenroeder PT, Hargens AR, Lundborg GN, Akeson WH: The carpal tunnel syndrome: a study of carpal canal pressure. J Bone Joint Surg $(A m)$ 1981;63-A: 380-383.

9. Redmond MD, Rivner MH: False positive electrodiagnostic tests in carpal tunnel syndrome. Muscle Nerve 1988;11: 511-517.

10. Masgaradeh M, Schneck CD, Bonakdarpour A, Mitra A, Conaway D: Carpal Tunnel: MR imaging part II. Carpal tunnel syndrome. Radiology 1989;171:749-754.

11. Nathan PA, Keniston RC, Myers LD, Meadows KD: Obesity as a risk factor for slowing of sensory conduction of the median nerve in industry: A cross-sectional and longitudinal study involving 429 workers. $J$ Occup Med 1992;34: $379-383$.

12. Szabo RM, Chidgey LK: Stress carpal tunnel pressures in carpal tunnel patients and normal patients. $J$ Hand Surg $1989 ; 15$ A:624-628.

13. STATA, Computing Resource Center, Santa Monica, CA, 1992.

14. Stevens JC, Sun S, Beard CM, O'Fallon WM, Kurland LT: Carpal tunnel syndrome in Rochester, Minnesota, 1961-1980. Neurology 1988;38:134-138.

15. Vessey MP, Villard-Mackintosh L, Yeates D: Epidemiology of carpal tunnel syndrome in women of childbearing age. Findings in a large cohort study. Int J Epidemiol 1990;19: $655-659$.

16. Werner CO, Elmqvist D, Ohlin P: Pressure and nerve lesion in the carpal tunnel. Acta Orthop Scand 1983;54:312-316.

17. Wieslander G, Norback D, Gothe CJ, Juhlin L: Carpal tunnel syndrome (CTS) and exposure to vibration, repetitive wrist movements, and heavy manual work: a case-referent study. Br J Ind Med 1989;46:43-47. 